\title{
Keine Vorteile durch Plasmapherese
}

Fragestellung: Die primäre Fragestellung der Studie war die retrospektive Untersuchung, ob Plasmapherese das Überleben und den klinischen Outcome bei Patienten mit Natalizumabassoziierter progressiver multifokaler Leukenzephalopathie (PML) verbessert.

Hintergrund: Natalizumab, ein anti-Integrin-a4 $\beta 1$ monoklonaler Antikörper zur Behandlung der hochaktiven schubförmig remittierenden Multiplen Sklerose, hemmt die Einwanderung von Immunzellen in das entzündete Zentralnervensystem (ZNS). Bis April 2017 wurden 714 PML-Fälle unter Natalizumab mit einer Mortalität von $24 \%$ berichtet; eine spezifische Therapie existiert nicht. Um die schnelle Wiederherstellung der Immunüberwachung im ZNS zu erzielen, wird aktuell empirisch die Beschleunigung der Elimination des Antikörpers mittels Plasmapherese und nicht das abwartende Procedere mit spontaner Erholung des Immunsystems empfohlen. Die

Landi D, De Rossi N, Zagaglia S et al. No evidence of beneficial effects of plasmapheresis in natalizumab-associated PML. Neurology 2017; 88: 1144-52
Immunrekonstitution macht sich allerdings oft mit einer klinisch relevanten Verschlechterung (Immunrekonstitutionssyndrom, IRIS) bemerkbar.
Patienten und Methodik: Insgesamt 184 Natalizumab-PMLFälle aus 49 zwischen 2005 bis 2015 in PubMed veröffentlichten Artikeln und 34 italienische Natalizumab-PML-Fälle wurden retrospektiv analysiert. Als primäre Outcome-Parameter wurden das Überleben und der klinische Status post PML analysiert.

Ergebnisse: Eine Plasmapherese wurde bei 184 (84\%) Patienten durchgeführt. Die zwei Gruppen (PLEX+ und PLEX-) zeigten signifikante Unterschiede für Geschlecht, JCV-Kopien im Liquor (Medianwert PLEX+ 340, PLEX- 57 Kopien) und PMLLäsionslokalisierung (87\% PLEX+, 68\% PLEX- mit supratentorieller Beteiligung). Sowohl die Mortalität als auch der klinische Outcome post-PML, aber auch die Entwicklung von IRIS waren in den beiden Gruppen ähnlich. Die Behandlung von PML-IRIS mit Steroiden führte dagegen zu einer reduzierten Mortalität.

Schlussfolgerungen: Die Autoren konnten keine klinischen Vorteile der Plasmapherese-Behandlung bei der aktuellen Kohorte feststellen. Diese Beobachtung könnte eine zukünftige individualisierte Beurteilung favorisieren, um die Patienten zu identifizieren, die eher von Plasmapherese in der NatalizumabPML-Behandlung profitieren.

\section{- Kommentar von Kalliopi Pitarokoili und Ralf Gold, Bochum}

\section{Retrospektive Studie mit reduzierter statistischer Aussagekraft}

In Ermangelung von Daten aus größeren Studien ist diese Analyse hilfreich, wenn auch von reduzierter statistischer Aussagekraft. Die Haupteinschränkungen beziehen sich auf die retrospektive Natur der Auswertung und die indirekte Erhebung der klinischen Maßnahmen aus den veröffentlichten Fällen.

Erstens bestanden zwischen den beiden Gruppen PLEX+ und PLEX-signifikante demografische (Geschlecht) oder PMLbezogene (supratentorielle Beteiligung, JCV-Kopien im Liquor) Unterschiede, die den Outcome beeinflussen. Ferner waren in die Gruppe PLEX-Patienten eingeschlossen, die vor allem aufgrund einer verspäteten Diagnose keine PLEX erhalten haben - drei Monate nach der letzten Natalizumab-Infusion ist das Medikament üblicherweise im Körper abgebaut. In diesem Fall besteht möglicherweise ein Selektions-Bias für Patienten mit guter Prognose trotz spätere Diagnosestellung.

Schließlich wurden aufgrund der begrenzten verfügbaren Informationen, Daten über PLEX-Protokolle, Intervall zwischen letzter Natalizumab-Gabe und PML-Diagnose und Schwere der IRIS nicht mitbeurteilt. Daher konnte man nicht abschätzen, ob die IRIS bei Patienten, die mit PLEX behandelt wurden, leichter war.

Trotz dieser Einschränkungen, konnte diese Studie positive prognostische Faktoren (jüngeres Alter und niedrigere JCV-
Kopien im Liquor bei PML-Diagnose, niedrigere EDSS-Scores vor PML) sowie die Effektivität der IRIS Behandlung mit Steroiden unterstützen.

In Anbetracht der hohen Mortalität und residualer Behinderung der Natalizumab-PML bleiben die rasche Diagnosestellung, die schnelle Wiederherstellung des Immunsystems und die Steroidtherapie der IRIS unseres Erachtens wichtige Aspekte der Therapieplanung. Unter Berücksichtigung der potenziellen Risiken von PLEX sind sicherlich individualisierte Entscheidungen über PLEX möglich bis zur Erweiterung von unseren Kenntnissen zur PML-Pathophysiologie.

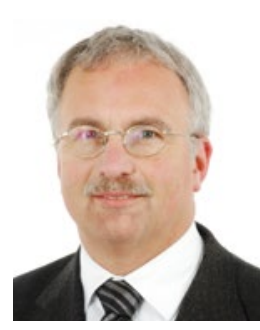

Prof. Dr. med. Ralf Gold, Bochum

St. Josef-Hospital, Ruhr-Universität Bochum Email: ralf.gold@rub.de 